\title{
Online Channel Allocation for Full-Duplex Device-to-Device Communications
}

\author{
Gilsoo Lee ${ }^{\dagger}$, Walid Saad ${ }^{\dagger}$, Mehdi Bennis ${ }^{\ddagger}$, Abolfazl Mehbodniya ${ }^{\S}$, and Fumiyuki Adachi ${ }^{\S}$ \\ $\dagger$ Wireless@VT, Bradley Department of Electrical and Computer Engineering, Virginia Tech, Blacksburg, VA, USA, \\ Emails: \{gilsoolee,walids\}@vt.edu. \\ ¥ Centre for Wireless Communications, University of Oulu, Finland, Email: bennis@ee.oulu.fi. \\ $\S$ Dept. of Communication Engineering, Graduate School of Engineering, Tohoku University, Sendai, Japan, \\ Emails: mehbod@mobile.ecei.tohoku.ac.jp,adachi@ecei.tohoku.ac.jp.
}

\begin{abstract}
Full-duplex device-to-device (D2D) communications over cellular networks is a promising solution for maximizing wireless spectral efficiency. However, in practice, due to the unpredictable arrival of D2D users, the base station (BS) must smartly allocate suitable channels to arriving D2D pairs. In this paper, the problem of dynamic channel allocation is studied for full-duplex D2D networks. In particular, the goal of the proposed approach is to maximize the system sum-rate under complete uncertainty on the arrival process of D2D users. To solve this problem, a novel approach based on an online weighted bipartite matching is proposed. To find the desired solution of the channel allocation problem, a greedy online algorithm is developed to enable the BS to decide on the channel assignment for each D2D pair, without knowing any prior information on future D2D arrivals. For an illustrative case study, upper and lower bounds on the competitive ratio that compares the performance of the proposed online algorithm to that of an offline algorithm are derived. Simulation results show that the proposed online algorithm can achieve a near-optimal sum-rate with an optimality gap that is no higher than $8.3 \%$ compared to the offline, optimal solution that has complete knowledge of the system.
\end{abstract}

\section{INTRODUCTION}

To cope with emerging mobile data traffic, device-to-device (D2D) communication has been proposed as a key technology for improving wireless capacity and coverage [1]. D2D over cellular networks allows wireless users to directly communicate with each other without using the cellular infrastructure thus reducing power consumption and improving data rates [2]. As D2D user pairs are typically within a short distance of one another, one can exploit the potential of full-duplex D2D communications to further improve the system performance. However, to reap the benefits of full-duplex D2D communications, one must address a number of challenges ranging from interference management to network modeling and resource allocation [3]-[8].

In D2D communications, D2D users coexist with cellular users and share their resources. Such resource sharing, if not properly managed, can lead to harmful mutual interference. Thus, a careful interference management scheme is required under the coordination of a base station (BS) [3]. To mitigate

This research been supported by the U.S. National Science Foundation under Grant CNS-1460333 and by Towards Energy-Efficient Hyper-Dense Wireless Networks with Trillions of Devices, the Commissioned Research of National Institute of Information and Communications Technology (NICT), Japan, and by the Academy of Finland. interference and enhance spectral efficiency, the works in [4][6] study several resource allocation strategies. For instance, the authors in [4] propose a resource allocation scheme to maximize the network throughput with quality-of-service (QoS) constraints. The authors in [5] propose a channel assignment algorithm based on dynamic programming. Then, a suboptimal clustering algorithm is proposed to form groups of users over a bipartite graph, and, then, a queueing-based algorithm is used to determine channel assignments for clusters. In [6], the authors develop a game-theoretic model to address the problem of D2D sum-rate maximization under QoS constraints.

Furthermore, the authors in [7] and [8] study spectrum resource sharing for full-duplex radios and D2D communications. The work in [7] investigates the problem of maximizing user connectivity by proposing a two-stage approach. In the first stage, a bipartite matching problem is used to assign halfduplex users to the channels of a full-duplex BS, and, then the remaining users are offloaded to the unlicensed bands by using D2D mode. In [8], the authors study the system performance when full-duplex or half-duplex D2D users share the cellular channels to maximize the system throughput.

In all of these existing D2D and full-duplex communication resource management works [3]-[8], it is generally assumed that information on the D2D users such as the total number and locations of the D2D users is completely known. However, in practice, D2D communication can be spontaneously initiated by users that dynamically join and leave the network and, as such, the presence of a D2D link can be uncertain. Indeed, it is challenging for the network to know when and where a D2D pair will be available. This is particularly important in dense networks in which users can join and leave at a high rate. Thus, there exists an inherent uncertainty stemming from the unknown locations or number of D2D users. Further, most of the existing works [4]-[6] on channel assignment problems typically assume half-duplex communications for D2D users. In contrast, the use of in-band full-duplex D2D users which can transmit and receive information simultaneouly over the same channel can significantly improve D2D performance, if properly deployed. Consequently, unlike the existing literature [4]-[6] which assumes full information knowledge for halfduplex D2D, our goal is to assign, using an online approach, the best channel for each full-duplex D2D users under uncer- 


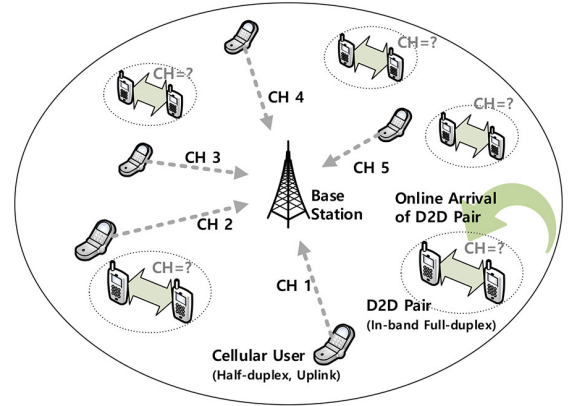

Fig. 1: System model of the underlaid D2D network in the cellular system.

tainty.

The main contribution of this paper is to develop a novel framework for online channel allocation in full-duplex D2D networks. This framework allows the network to dynamically allocate the most suitable channel to newly arriving D2D users in the presence of uncertainty on the arrival order of D2D pairs. In particular, we formulate an online optimization problem whose objective is to maximize the sum data rate of all D2D users by properly assigning channels. To solve this problem, we propose novel approach based on the tools of online weighted bipartite matching. We solve the proposed online matching problem using a practical greedy algorithm that enables each D2D pair to smartly share the channels that are already used by cellular users without any prior information on future D2D arrivals and their locations. For an illustrative case study, we derive upper and lower bounds on the competitive ratio to compare the performance of the proposed online algorithm to that of an offline algorithm. Simulation results show that the proposed online algorithm can maximize the sum data rate of the D2D network and achieve a performance that is near-optimal compared to an offline solution that has full information on D2D arrivals.

The rest of this paper is organized as follows. In Section II, the system model is presented. In Section III, we formulate the proposed online problem. Section IV presents our proposed online solution. Simulation results are analyzed in Section V while conclusions are drawn in Section VI.

\section{SySTEM MOdEL}

Consider a cellular network in which cellular users share resources with an underlaid, full-duplex D2D network. As shown in Fig. 1, $M$ cellular users are present within the coverage area of a single BS. Each cellular user $m \in \mathcal{M} \triangleq$ $\{1, \cdots, M\}$ uses a channel of bandwidth $B$. We consider an OFDMA system in which the total number of channels in the system is equal to $M$. Since each channel is allocated to each user, we use the same index $m$ for cellular user and the user's channel. We assume that the cellular network is fully loaded [4] such that all $M$ cellular users occupy the $M$ orthogonal channels without leaving any spare spectrum. Without loss of generality, we consider that this spectrum sharing occurs during the uplink of cellular transmissions. Under this singlecell OFDMA model, cellular users will not interfere with each other. Moreover, the BS and non-D2D cellular users are assumed to operate in half-duplex mode as typically done in the literature [8].

In our network, $M$ cellular users coexist with $I$ D2D pairs. The pairing of D2D users is assumed to be pre-determined. The total number of the D2D users is $2 I$. Each pair of $\mathrm{D} 2 \mathrm{D}, i \in \mathcal{I} \triangleq\{1, \cdots, I\}$, consists of two users denoted by $2 i-1$ and $2 i$, respectively. While the cellular users use half-duplex communications, all D2D users exploit full-duplex communications [8]. Since the D2D-paired UEs can transmit and receive information over the same channel, if properly optimized, full-duplex communication can increase the data rate of the D2D network. In our model, given their proximity, the D2D users can use a lower transmit power than the cellular users to mitigate the interference to other cellular users and D2D pairs.

First, we define the uplink data rate of a cellular user $m$ where a cellular user and D2D pair $i$ share channel $m$. The transmit powers of a cellular user and a D2D user are, respectively, denoted by $P^{C}$ and $P^{D}$. In the uplink of cellular user $m$, the BS experiences interference over channel $m$ due to the transmission of the D2D pair that is sharing channel $m$. Therefore, when cellular user $m$ transmits information to the BS, the signal-to-interference-plus-noise ratio (SINR) over channel $m$ at the BS will be:

$$
\Gamma_{i}^{C}(m)=\frac{g_{m} P^{C}}{B N_{0}+\sum_{k=\{2 i-1,2 i\}} g_{k} P^{D}},
$$

where $N_{0}$ is the noise spectral density, $g_{m}=\beta d_{m}^{-\alpha}$ is the channel gain with $d_{m}$ being the distance between cellular user $m$ and the BS, and $g_{k}=\beta d_{k}^{-\alpha}$ is the channel gain between D2D user $k$ and the BS with $d_{k}$ being the distance between them. $\alpha$ and $\beta$ are, respectively, the path loss exponent and path loss constant. We assume perfect channel state information is available. Also, a time-invariant block fading channel is considered. Then, the uplink data rate of a cellular user $m$ will be:

$$
R_{i}^{C}(m)=B \log _{2}\left(1+\Gamma_{i}^{C}(m)\right) \text {. }
$$

Thus, if the received signal power from the D2D users increases, the cellular user experiences a higher co-channel interference during the uplink. To effectively share the cellular channels, the D2D users must ensure that the cellular users' QoS does not go below a certain threshold. When assigning the channels for D2D users, the data rate of existing cellular users must be maintained at a minimum threshold $\gamma^{C}$ so that the QoS is guaranteed. To share a channel $m$ with a D2D pair $i$, the data rate of the cellular user has to be greater than the threshold $\gamma^{C}$ such that $R_{i}^{C}(m) \geq \gamma^{C}$. We define a variable $\omega_{i, m}$ to indicate whether a D2D pair $i$ can be assigned to a channel $m$ as follows:

$\omega_{i, m}=\left\{\begin{array}{lc}1, & \text { if D2D pair } i \text { is admissible to channel } m, \\ 0, & \text { otherwise. }\end{array}\right.$

To measure the performance of the D2D network, we define the data rate of D2D pair $i$. When two D2D-paired users $k=$ 
$2 i-1$ and $k^{\prime}=2 i$ share a channel $m$ with cellular user $m$, the SINR of a D2D link from user $k$ to $k^{\prime}$ will be:

$$
\Gamma_{k \rightarrow k^{\prime}}(m)=\frac{g_{k, k^{\prime}} P^{D}}{B N_{0}+g_{m, k^{\prime}} P^{C}+g_{0} g_{k^{\prime}, k^{\prime}} P^{D}},
$$

where $g_{k, k^{\prime}}=\beta d_{k, k^{\prime}}^{-\alpha}$ is the channel gain with $d_{k, k^{\prime}}$ being the distance between D2D users $k$ and $k^{\prime}, g_{m, k}=\beta d_{m, k}^{-\alpha}$ is the channel gain between D2D user $k$ and the cellular user $m$ with $d_{m, k}$ being the distance between them, $g_{0}$ is the selfinterference cancellation at the analog components, and $g_{k^{\prime}, k^{\prime}}$ shows the self interference. Similarly, we define $\Gamma_{k^{\prime} \rightarrow k}(m)$ as the SINR of a D2D link from user $k^{\prime}$ to $k$. Due to channel reciprocity, we assume that $g_{k, k^{\prime}}$ equals to $g_{k^{\prime}, k}$. Then, the data rate in D2D mode will be given by:

$R_{i}^{D}(m)=B\left(\log _{2}\left(1+\Gamma_{k \rightarrow k^{\prime}}(m)\right)+\log _{2}\left(1+\Gamma_{k^{\prime} \rightarrow k}(m)\right)\right)$.

The sum data rate of the uplink of users $k$ and $k^{\prime}$ captures the data rate of the $\mathrm{D} 2 \mathrm{D}$ pair $i$ due to the full-duplex communications. In (5), we observe that the uplink of a cellular user will create interference at the corresponding D2D thus affecting its data rate.

\section{PROBLEM FORMULATION}

Given the defined system model, our goal is to analyze the optimal channel assignment problem for D2D users. In the D2D network, D2D links can be created in a dynamic manner as users join and leave the network. As such, the BS is unable to know a priori whether new D2D pairs will be formed in the network or not. Moreover, since the total number of D2D pairs as well as the location of each such pair are unknown and highly unpredictable, optimizing channel assignment becomes quite challenging. Under such uncertainty, assigning channels to existing D2D pairs must also account for potential arrival of new D2D pairs. In fact, even if a given channel allocation can improve the performance of an existing D2D pair, it may have a detrimental effect on an incoming pair. To cope with the uncertainty of D2D arrivals while considering the data rate of current and future D2D pairs, we introduce an online optimization scheme that can handle channel assignment under uncertainty.

First, we formulate the following online channel assignment problem whose goal is to maximize the sum data rate of all D2D users:

$$
\begin{aligned}
\max _{\boldsymbol{x}} & \sum_{i=1}^{I} \sum_{m=1}^{M} x_{i, m} R_{i}^{D}(m), \\
\text { s.t. } & \sum_{m=1}^{M} x_{i, m} \leq 1, \forall i \in \mathcal{I}, \\
& \sum_{i=1}^{I} x_{i, m} \leq 1, \forall m \in \mathcal{M}, \\
& R_{i}^{C}(m) \geq \gamma^{C},
\end{aligned}
$$

where $\boldsymbol{x}$ is a vector whose elements $x_{i, a}, \forall i \in \mathcal{I}, m \in \mathcal{M}$, indicate the channel assignment. In (6), the objective function is the sum of data rate of all current D2D users. We determine the channel assignment $\boldsymbol{x}$ so that the sum data rate is maximized. To guarantee the QoS of cellular users, if $\omega_{i, m}=0$, then $x_{i, m}=0$.

In (6), while the number of channels and information about cellular users are known, we assume that D2D pairs arrive in an online and arbitrary manner. This implies that the information about each D2D pair is collected sequentially. For example, a couple of non-D2D users can be paired spontaneously to initiate a D2D link. Similarly, D2D-paired users that were idle can suddenly re-initiate a D2D link. Both such cases show that the BS is unable to know any information on future D2D pairs. Therefore, in our problem, the arrival order is represented by an index $i$. At each arrival event, the arrival order $i$ increases by one. Also, index $i$ can be seen as the time order of arrival. Thus, the first arriving D2D pair is indexed by $i=1$, and similarly, the D2D pair that arrives at order $n$ has index $i=n$. The number of D2D pairs is unpredictable, and, thus, $I$ is an unknown value. When D2D pair $i=n$ arrives, we know the information of only the D2D pairs $i \leq n$. Under such incomplete information, finding the optimal solution of (6) is challenging and, as such, one has to seek an online, sub-optimal solution that is robust to uncertainty.

When a D2D pair appears in the network, we must assign it one of the cellular channels. Since each channel has a limited capacity, the channel allocated to a given D2D pair may be re-allocated to another, incoming D2D pair if this newly arriving pair can yield a higher rate. The first constraint of problem (6) shows that one or no channel can be assigned to the D2D pair $i$. This also implies that, if a D2D pair $i$ does not acquire a channel, then this pair cannot communicate. The second constraint indicates the a cellular user's channel $m$ can be shared with at most one D2D pair. Note that, if the number of D2D pairs $I$ is greater than the number of channels $M$, the proposed solution can still be applied as follows. When the first $M \mathrm{D} 2 \mathrm{D}$ pairs are assigned to $M$ channels, the next $K$ arriving D2D pairs can be assigned by considering the interference from not only cellular users but also newly arriving D2D users. In this case, constraint (7) can be substituted by $\sum_{m=1}^{M} x_{i, m} \leq\lceil I / M\rceil$.

The dual of problem (6) will be:

$$
\begin{aligned}
\min _{\boldsymbol{\mu}, \boldsymbol{\lambda}} & \sum_{m=1}^{M} \mu_{m}+\sum_{i=1}^{I} \lambda_{i}, \\
\text { s.t. } & \mu_{m}+\lambda_{i} \geq R_{i}^{D}(m), \forall m \in \mathcal{M}, \forall i \in \mathcal{I}, \\
& \mu_{m} \geq 0, \lambda_{i} \geq 0, \forall m \in \mathcal{M}, \forall i \in \mathcal{I}, \\
& R_{i}^{C}(m) \geq \gamma^{C},
\end{aligned}
$$

where vectors $\boldsymbol{\mu}$ and $\boldsymbol{\lambda}$ consist of elements $\mu_{m}, \forall m \mathcal{M}$, and $\lambda_{i}, \forall i \in \mathcal{I}$, respectively. In (10), the dual solutions are $\boldsymbol{\mu}$ and $\boldsymbol{\lambda}$. We use an online algorithm to solve (6) and (10) to optimize the channel assignment in Section IV. 


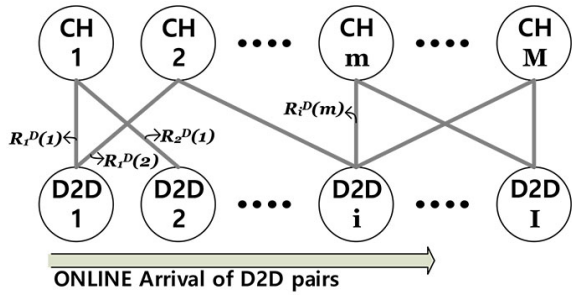

Fig. 2: Illustration of constructing a weighted bipartite graph.

\section{Online Channel Assignment: Weighted BIPARTITE MATCHING APPROACH}

\section{A. Construction of a Weighted Bipartite Graph}

To represent the online problem on a graph, we build a weighted bipartite graph $G=(\mathcal{U}, \mathcal{V}, \mathcal{E})$ in which the cellular channels are denoted by the vertex set $\mathcal{U}$, the D2D users are captured by the vertex set $\mathcal{V}$, and the achievable data rates of each D2D pair are represented by the set of edges $\mathcal{E}$. For example, in Fig. 2, a total of $M$ channels are shown as vertices in the upper half, and the D2D pairs are shown in the lower half. Also, the edges show the data rate of D2D pair $i$ using channel $m$, i.e., $R_{i}^{D}(m)$. If $\omega_{i, m}=0$, the two vertices $i$ and $m$ are not connected. Every edge indicates that a matching between D2D pair $i$ and channel $m$ is possible. Note that the D2D pairs arrive online. Thus, for example, when D2D pair 1 arrives in Fig. (2), we do not have any information on any D2D pair $i \geq 2$. When a D2D arrival event happens, the related values including $\omega_{i, m}$ and $R_{i}^{D}(m), \forall i \in \mathcal{I}, \forall m \in \mathcal{M}$ are calculated, and the graph is updated.

\section{B. Greedy Online Algorithm: Procedure and Analysis}

To find the channel assignment vector $\boldsymbol{x}$ that maximizes the sum rate, we propose a greedy online algorithm shown as Algorithm 1. The BS can use Algorithm 1 to assign channels to each D2D pairs. To develop the proposed algorithm, we use the structure of the dual problem (10). Our proposed greedy algorithm builds on the primal-dual algorithm with greedy update rule that is introduced in [9].

In Algorithm 1, we first initialize the dual variable $\mu_{m}$ to 0 for all channels $m$. When a D2D pair $i$ arrives online, we calculate the gain that is defined by $R_{i}^{D}(m)-\mu_{m}$ for all channels. After that, the channel $m^{*}$ that has the largest gain is assigned to the D2D pair $i$, and we set $x_{i, m^{*}}=1$. If the gain for each channel is negative, then we do not assign a channel and leave the D2D pair unassigned. Also, if channel $m^{*}$ has been already assigned to another D2D pair $i^{\text {old }}$, then the previous channel assignment of $i^{\text {old }}$ is canceled; thus, we set $x_{i_{\text {old }}^{\text {ol }}, a^{\prime}}=0$. Finally, we can have the dual solutions, $\lambda_{i}$ and $\mu_{m *}$. We update $\lambda_{i}=R_{i}^{D} m^{*}-\mu_{m^{*}}$. Here, we set $\beta_{m^{*}}$ to the value of $R_{i}^{D}\left(m^{*}\right)$. Essentially, for each $i$, we find the solution by increasing $\mu_{m}$ and calculating the corresponding $\lambda_{i}$ while $\mu_{m}$ and $\lambda_{i}$ should satisfy the constraint (11). The complexity of Algorithm 1 experienced by a D2D pair at each time pertains to the process of finding the maximum value in an array, so the worst-case complexity is $\mathcal{O}(M)$.

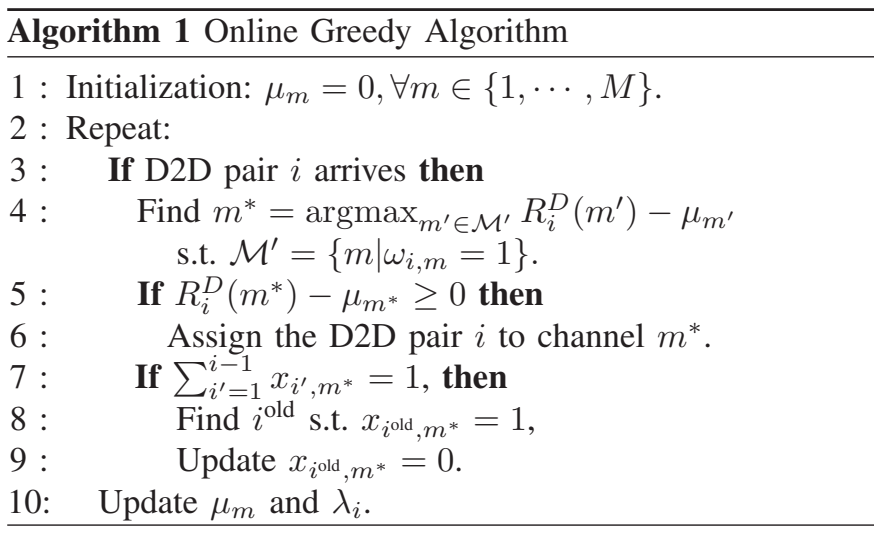

Since the size of the search space is limited by the number of channels, Algorithm 1 can be executed in a reasonably short time. The online algorithm significantly reduce the complexity compared to the offline, exhaustive search approach which has a complexity of $\mathcal{O}\left(2^{M}\right)$.

To illustrate the effect of reassigning a channel, we consider the case in which a new D2D pair $i^{\text {new }}$ arrives, and the BS reassigns channel $m$ from $i^{\text {old }}$ to $i^{\text {new }}$. This will only happen if the data rate of $i^{\text {new }}$ is greater than $\mu_{m}$. It is due to the fact that $\lambda_{i^{\text {new }}}=R_{i^{\text {new }}}^{D}(m)-\mu_{m} \geq 0$ where $\mu_{m}=R_{i^{\text {old }}}^{D}$. Thus, we have $R_{i^{\text {new }}}^{D}(m) \geq \mu_{m}=R_{i^{\text {old }}}^{D}$. Consequently, the proposed algorithm always find the channel assignment that has incremental, marginal gain; the sum data rate of the D2D network increases at each D2D arrival event unless the D2D pair that arrived is assigned to a channel.

While for the general case with $M$ D2D users, analytical results are challenging to derive, we can still gain an insight on the algorithm performance for a special case with two channels and two D2D pairs arriving online. For this two D2D pairs, two channels case, we assume that D2D pair 1 arrives first where D2D pair 1 can be assigned to channel 1 and 2. Then, D2D pair 2 arrives, but it can be assigned to channel 1 only. This example is illustrated in Fig. 2 if we account for D2D pair 1 and 2 only. Then, for the given example, we provide an analysis to measure the benefit of the proposed online algorithm. For the analysis, we define $A L G$ and $O P T$. $A L G$ is the value of the objective function when using the proposed algorithm while $O P T$ is the optimal value of the problem (6) when using an offline solution. The offline scenario means that we already know all the information on the total number, the locations, and achievable data rates of D2D pairs. To measure the performance of the proposed online algorithm, we compare the ratio between $A L G$ and $O P T$. This is known as competitive analysis, and a ratio $\frac{A L G}{O P T}$ is called a competitive ratio. A competitive ratio can be a metric showing how close the performance of an online algorithm is compared to an offline optimal solution.

For our case study, we derive the upper and lower bounds on the competitive ratio to compare the performance of the proposed algorithm compared to the optimal solution as follows. 
Theorem 1. We show that a ratio between $O P T$ and $A L G$ satisfies $\frac{1}{2}<\frac{A L G}{O P T}<1$ for the given example.

Proof. When D2D pair 1 arrives, D2D pair 1 can be assigned to channel 1 or 2 where channel 1 and 2 yields different data rates, $R_{1}^{D}(1)$ and $R_{1}^{D}(2)$, respectively. First, if we consider $R_{1}^{D}(1)<R_{1}^{D}(2)$, then D2D pair 2 is assigned to channel 1 , thus having data rate $R_{2}^{D}(1)$. In this case, the channel assignment by the online algorithm is also optimal. Thus, we have $A L G=O P T=R_{1}^{D}(2)+R_{2}^{D}(1)$, and $\frac{A L G}{O P T}=1$.

Next, we consider the other case $R_{1}^{D}(1)>R_{1}^{D}(2)$. Then, the online algorithm assigns D2D pair 1 to channel 1. After that, D2D pair 2 arrives where it can use channel 1 only. However, channel 1 has been assigned to D2D pair 1 due to $R_{1}^{D}(1)>R_{1}^{D}(2)$. If $R_{2}^{D}(1)-\mu_{1}=R_{2}^{D}(1)-R_{1}^{D}(1)>0$, then channel 1 is reassigned to D2D pair 2, and D2D pair 1 loses channel assignment. In this case, the results are $A L G=$ $R_{2}^{D}(1)$ and $O P T=R_{1}^{D}(2)+R_{2}^{D}(1)$. Since $R_{2}^{D}(1)>R_{1}^{D}(1)$ and $R_{1}^{D}(1)>R_{1}^{D}(2)$, we have inequalities given by $\frac{1}{2}=$ $\frac{R_{2}^{D}(1)}{R_{2}^{D}(1)+R_{2}^{D}(1)}<\frac{R_{2}^{D}(1)}{R_{2}^{D}(1)+R_{1}^{D}(1)}<\frac{R_{2}^{D}(1)}{R_{2}^{D}(1)+R_{1}^{D}(2)}$. Moreover, due to $R_{1}^{D}(1)<R_{2}^{D}(1)<R_{2}^{D}(1)+R_{1}^{D}(2)$, we additionally know $\frac{R_{2}^{D}(1)}{R_{2}^{D}(1)+R_{1}^{D}(2)}<1$. Therefore, the boundaries of the ratio can be shown as

$$
\frac{1}{2}<\frac{A L G}{O P T}=\frac{R_{2}^{D}(1)}{R_{1}^{D}(2)+R_{2}^{D}(1)}<1 .
$$

Otherwise, channel 1 is still assigned to D2D pair 1 , and D2D pair 2 do not acquire a channel. Then, the result are $A L G=R_{1}^{D}(1) ; O P T=R_{1}^{D}(1)$ if $R_{1}^{D}(1)>R_{1}^{D}(2)+R_{2}^{D}(1)$, and otherwise, $O P T=R_{1}^{D}(2)+R_{2}^{D}(1)$. If $O P T=A L G=$ $R_{1}^{D}(1)$, then $\frac{A L G}{O P T}=1$. Thus, we focus on the later case $O P T=R_{1}^{D}(2)+R_{2}^{D}(1)$ when $R_{1}^{D}(1)<R_{1}^{D}(2)+R_{2}^{D}(1)$. In this case, we have two conditions, $R_{1}^{D}(1)>R_{1}^{D}(2)$ and $R_{1}^{D}(1)>R_{2}^{D}(1)$, so the two inequalities result in $2 R_{1}^{D}(1)>$ $R_{1}^{D}(2)+R_{2}^{D}(1)$ that is $\frac{1}{2}<\frac{R_{1}^{D}(1)}{R_{1}^{D}(2)+R_{2}^{D}(1)}$. Therefore, the competitive ratio can be bounded a follows:

$$
\frac{1}{2}<\frac{A L G}{O P T}=\frac{R_{1}^{D}(1)}{R_{1}^{D}(2)+R_{2}^{D}(1)}<1 .
$$

Hence, in the given example of the online maximization problem, the value achieved by the online algorithm is at least a half of the offline optimal value.

For the more general case, we will provide thorough analysis via simulations in Section V.

\section{Simulation Results}

For our simulations, we consider a single-cell environment where the cellular users are uniformly distributed within a $50 \mathrm{~m} \times 50 \mathrm{~m}$ area. We consider a network with 10 channels each of which is allocated to a cellular user. Also, the sequence of D2D users' arrival follows a uniform distribution. The bandwidth of each channel is $200 \mathrm{kHz}$, such that the total bandwidth of the single cell is $2 \mathrm{MHz}$. The power spectral density of the thermal noise is $-174 \mathrm{dBm} / \mathrm{Hz}$. We

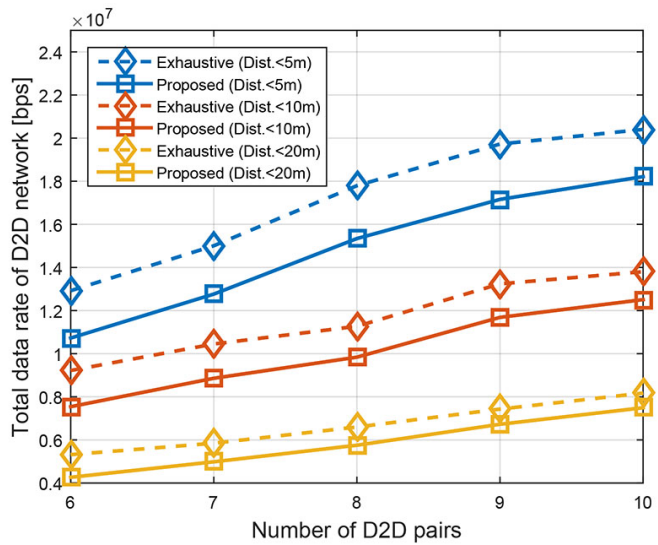

Fig. 3: The total data rate of the D2D network compared with optimal, offline algorithm for the different maximum D2D distances.

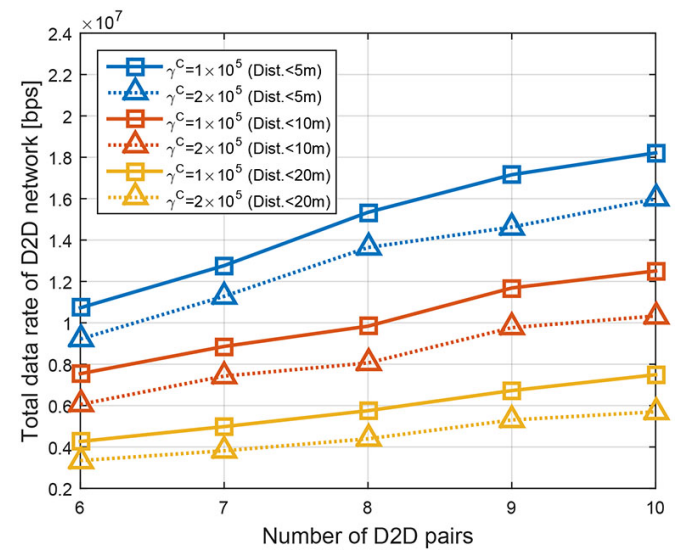

Fig. 4: The total data rate of the D2D network for the different QoS thresholds of cellular users.

set $\alpha=2$ and $\beta=10^{-3}$ to model the channel gain. Also, at the reference distance of $1 \mathrm{~m}$, the channel gain is $-30 \mathrm{~dB}$ [10]. For the D2D users, $g_{k, k}$ is set to 0.03 , $\forall k \in\{2 i-1,2 i \mid \forall i \in \mathcal{I}\}$ assuming that the circulator provides $15 \mathrm{~dB}$ of isolation of self-interference [10], and we assume that an analog cancellation additionally provides $g_{0}=-60 \mathrm{~dB}$ of self-interference cancellation. Also, the location of D2D users are also uniformly distributed, and the distance between the two paired D2D users are less than the given maximum D2D distance. All statistical results are averaged over a large number of simulation runs. For comparison, we use the offline, optimal algorithm that has complete knowledge of the system.

In Fig. 3, we show the total data rate of the D2D network as the number of D2D pairs varies for various maximum D2D distances with $\gamma^{C}=1 \times 10^{5}$ bps. From this figure, we can first see that that the total data rate of our proposed algorithm achieves a performance that is quite close to the optimal solution derived using exhaustive search. This demonstrates the effectiveness of the proposed algorithm. For instance, Fig. 3 shows that the optimality gap is at most $8.3 \%$ at $20 \mathrm{~m}$ of the maximum D2D distance for a system with 10 D2D pairs. 


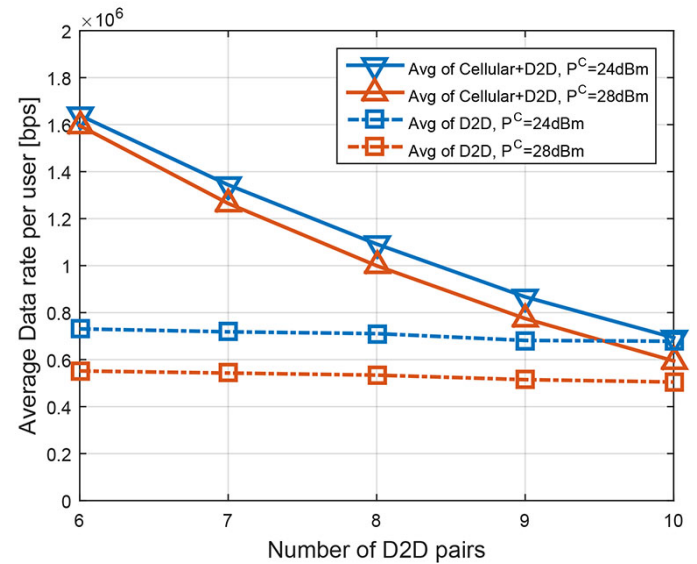

Fig. 5: The average data rate per user when cellular users use different transmit power.

In Fig. 4, we show the total data rate of the D2D network resulting from our proposed approach for different thresholds $\gamma^{C}$. From Fig. 4, we can see that, as the threshold value decreases, the overall data rate of the D2D network will increase. This is due to the fact that having a smaller threshold value will enable a D2D pair to have a larger number of channel options that can be assigned. This, in turn, prevents cases in which a D2D pair is not assigned to any channel. This can be commonly observed at all different D2D distances. For example, if the threshold $\gamma^{C}$ decreases from $2 \times 10^{5}$ to $1 \times 10^{5}$, the system exhibits a performance improvement of up to $31.4 \%$ in the total data rate at $10 \mathrm{D} 2 \mathrm{D}$ pairs and $20 \mathrm{~m}$ of the maximum D2D distance.

In Fig. 5, we show the average data rate of a user with $\gamma^{C}=1 \times 10^{5}$ bps and a maximum D2D distance of $10 \mathrm{~m}$, for two different transmit powers for the cellular users, 24 and $28 \mathrm{dBm}$. The results show that the D2D and total data rates increase when a lower transmit power is used by the cellular users. From Fig. 5, we also observe that the data rate of a user in the network decreases as the number of D2D pairs increases. This is due to the fact that an increase in the number of D2D pairs will naturally increase the interference on the cellular users. However, if a lower transmit power is used, then we can mitigate the effect of this interference at the expense of a lower rate for the cellular users. For instance, at 10 D2D pairs, the case using $24 \mathrm{dBm}$ of transmit power results in $34.3 \%$ higher average data rate for $\mathrm{D} 2 \mathrm{D}$ user compared to one using $28 \mathrm{dBm}$.

Fig. 6 shows the total number of the non-assigned D2D pairs for various maximum D2D distances. The proposed online algorithm results in a lower number of non-assigned D2D pairs when the maximum D2D distance decreases. This illustrates that as the distance between two D2D users becomes closer, the D2D pair may have larger number of channels where the D2D pair can be assigned. For example, Fig. 6 shows that decreasing the maximum D2D distance from $20 \mathrm{~m}$ to $5 \mathrm{~m}$ yields $19.6 \%$ reduction in the number of nonassigned D2D users.

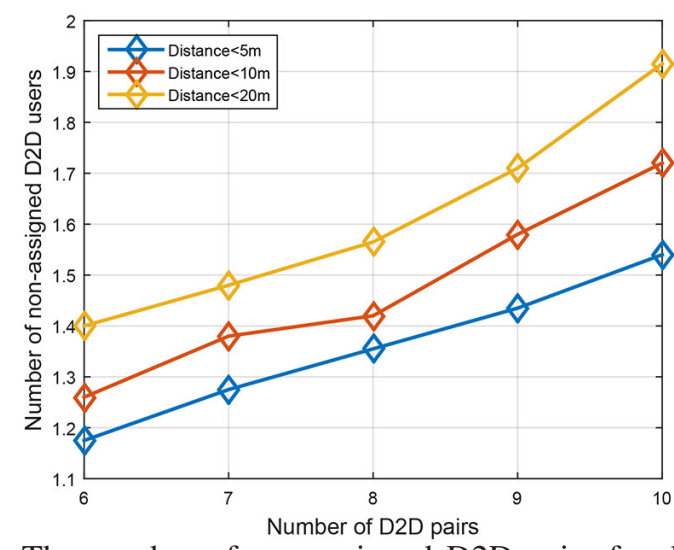

Fig. 6: The number of non-assigned D2D pairs for different maximum D2D distances.

\section{CONCLUSION}

In this paper, we have proposed a novel approach to optimize the channel assignment for the D2D network as an underlay of the cellular network. We have formulated the problem as an online weighted bipartite matching which enables the BS to assign channels to the D2D network effectively in the presence of uncertainty about D2D arrivals. We have shown that by using the greedy online algorithm, the suitable channel can be assigned to each D2D pair without knowing any prior information on future D2D arrivals. Simulation results have shown that the proposed online algorithm can achieve a total sum-rate that is no less than $8.3 \%$ below the optimal, offline solution found via exhaustive search.

\section{REFERENCES}

[1] K. Doppler, M. Rinne, C. Wijting, C. B. Ribeiro, and K. Hugl, "Deviceto-device communication as an underlay to LTE-Advanced networks," IEEE Commun. Mag, vol. 47, no. 12, pp. 42-49, Dec. 2009.

[2] G. Fodor, E. Dahlman, G. Mildh, S. Parkvall, N. Reider, G. Miklós, and Z. Turányi, "Design aspects of network assisted device-to-device communications," IEEE Commun. Mag, vol. 50, no. 3, pp. 170-177, Mar. 2012.

[3] F. Wang, L. Song, Z. Han, Q. Zhao, and X. Wang, "Joint scheduling and resource allocation for device-to-device underlay communication," in Proc. IEEE Wireless Commun. and Net. Conf., Shanghai, China, Apr. 2013, pp. 134-139.

[4] D. Feng, L. Lu, Y. Yuan-Wu, G. Y. Li, G. Feng, and S. Li, "Deviceto-device communications underlaying cellular networks," IEEE Trans. on Commun., vol. 61, no. 8, pp. 3541-3551, June 2013.

[5] R. Wang, J. Zhang, S. Song, and K. Letaief, "QoS-aware channel assignment for weighted sum-rate maximization in D2D communications," in Proc. of IEEE Global Commun. Conference (GLOBECOM), San Diego, CA, USA, Dec. 2015, pp. 1-6.

[6] Y. Gu, Y. Zhang, M. Pan, and Z. Han, "Cheating in matching of device to device pairs in cellular networks," in Proc. of IEEE Global Commun. Conf. (GLOBECOM), Austin, TX, USA, Dec. 2014, pp. 4910-4915.

[7] J.-W. Kao, Y.-Y. Shih, A.-C. Pang, and Y.-C. Lin, "Radio resource allocation for D2D-assisted full-duplex cellular networks," in Intl. Conf. on Ubq. and Future Netw., Sapporo, Japan, July 2015, pp. 721-726.

[8] S. Ali, N. Rajatheva, and M. Latva-aho, "Full duplex device-to-device communication in cellular networks," in Proc. of European Conf. on Net. and Commun. (EuCNC), Athens, Greece, Feb. 2014, pp. 1-5.

[9] A. Mehta, "Online matching and ad allocation," Theoretical Computer Science, vol. 8, no. 4, pp. 265-368, 2012.

[10] H. Ju, K. Chang, and M.-S. Lee, "In-band full-duplex wireless powered communication networks," in 17th Intl. Conf. on Adv. Commun. Technol. (ICACT), Gangwon, South Korea, July 2015, pp. 23-27. 